\title{
A very high accuracy potential energy surface for $\mathrm{H}_{3}$
}

\author{
Y.-S. Mark Wu ${ }^{a}{ }^{a}$ Aron Kuppermann ${ }^{a *}$ and James B. Anderson ${ }^{b}$ \\ ${ }^{a}$ A. A. Noyes Laboratory of Chemical Physics, Division of Chemistry and Chemical Engineering, \\ California Institute of Technology, Pasadena, CA 91125, USA \\ ${ }^{b}$ Department of Chemistry, The Pennsylvania State University, University Park, PA 16802, USA
}

\author{
Received 10th November 1998, Accepted 22nd December 1998
}

\begin{abstract}
An exact quantum Monte Carlo (EQMC) method was used to calculate the potential energy surface (PES) for the ground electronic state of $\mathrm{H}_{3}$ over a grid of about 76000 nuclear geometries. The absolute ab initio statistical or sampling error of the calculation was $\pm 0.01 \mathrm{kcal} \mathrm{mol}^{-1}$ for energies $(V)$ smaller than $3 \mathrm{eV}$. This PES was fitted by a three-dimensional cubic spline method and the fitting accuracy was determined from a set of 3684 randomly selected nuclear geometries not used in the fitting. For the range $V \leqslant 3 \mathrm{eV}$ the rms fitting error was $\pm 0.010 \mathrm{kcal} \mathrm{mol}^{-1}$, and the absolute value of the corresponding maximum error was $0.018 \mathrm{kcal}$ $\mathrm{mol}^{-1}$. This fitted EQMC PES is an order of magnitude more accurate than the best PES previously obtained for this system. Detailed comparisons are made with previous PESs, for the more dynamically important nuclear configurations.
\end{abstract}

\section{Introduction}

Three $a b$ initio fitted potential energy surfaces (PESs) for the ground electronic state of $\mathrm{H}_{3}$ and its isotopomers have been used in most of the calculations performed in the last decade on the quantum reaction dynamics of this system, ${ }^{1-16}$ the LSTH,${ }^{17,18}$ DMBE, ${ }^{19}$ and BKMP2 ${ }^{20}$ surfaces. All three have an estimated $a b$ initio and fitting accuracy of about $0.2 \mathrm{kcal}$ $\mathrm{mol}^{-1}$ and give reaction cross section results that are generally in good agreement with each other, justifying the label of "chemically accurate" for such PESs. One significant exception to this agreement occurs, however, under resonance conditions. Recent calculations of the differential cross-section of the reaction $\mathrm{H}+\mathrm{D}_{2}(v=0, j=0) \rightarrow \mathrm{HD}\left(v^{\prime}=0, j^{\prime}\right)+\mathrm{D}$ in the total energy range $E=1.40-1.60 \mathrm{eV}$ (measured with respect to the bottom of the isolated diatom well), using the LSTH PES, and including the effect of the geometric phase (GP), have shown the existence of a pronounced backward peak for $j^{\prime}=5$, having a fwhm of $41 \mathrm{meV} \cdot{ }^{14}$ A collision lifetime analysis of the scattering wave function furnished a resonance energy $E_{\text {res }}=1.497 \mathrm{eV}$ and a resonance lifetime of $164 \mathrm{fs}$. These calculations were repeated using the BKMP2 PES, whose barrier height is $9.61 \mathrm{kcal} \mathrm{mol}^{-1}$, only $0.19 \mathrm{kcal} \mathrm{mol}^{-1}$ lower than the LSTH one. The corresponding resonance energy, however, shifted downwards by $1.27 \mathrm{kcal} \mathrm{mol}^{-1} 15$ and the lifetime dropped to $52 \mathrm{fs}$, showing how very sensitive the resonance characteristics are to the details of the PES. Since in general the position of a pronounced peak can be determined to an energy within about $1-2 \%$ of its fwhm, it is estimated that changes in the saddle point region of the order of $\pm 0.015 \mathrm{kcal} \mathrm{mol}^{-1}$ could affect those characteristics in a detectable manner. As a result, in order to predict the resonance energy within experimental accuracy, a PES with an absolute accuracy of about $\pm 0.015 \mathrm{kcal} \mathrm{mol}^{-1}$ in the strong interaction region is probably necessary. Such a prediction is highly desirable to help guide experiments aimed at the detection of this important feature of reaction dynamics. This importance derives from the high sensitivity that resonances have to the PES, holding out the hope that an experimental measurement of their properties can lead to the determination of important features of the PES not achievable by other methods.
For these reasons, we decided to calculate the PES of $\mathrm{H}_{3}$ with an absolute accuracy of $\pm 0.01 \mathrm{kcal} \mathrm{mol}^{-1}$ and a fitting accuracy of $\pm 0.015 \mathrm{kcal} \mathrm{mol}^{-1}$ over a wide region of nuclear configuration space. The only method currently capable of achieving such an absolute accuracy is the exact quantum Monte Carlo (EQMC) method developed recently by Anderson and coworkers. ${ }^{21-26}$ This method was used by Diedrich and Anderson to calculate the $\mathrm{H}_{3} \mathrm{PES}$ at 378 points with very high accuracy, most of them (253) for collinear configurations. This number is insufficient to span the entire threedimensional (3D) surface and does not yield a 3D fit accurate enough to permit the scattering calculations that were desired. The results of the present extended calculations, their fitting, and a comparison with the LSTH and BKMP2 surfaces are presented. The EQMC method and the numerical parameters used are summarized in Section 2, and the design of the nuclear geometry grid and the fitting procedure are described in Section 3. The results and their discussion are given in Sections 4 and 5, respectively, and a summary in Section 6.

\section{Exact quantum Monte Carlo methodology and numerical parameters}

The EQMC methodology has been reviewed in some detail not too long ago, ${ }^{26}$ and only a short summary is presented here. It involves a Green's function quantum Monte Carlo procedure coupled with an exact cancellation scheme for imposing the appropriate antisymmetry condition on the ground state wave function. A trial wave function is selected having this antisymmetry, and a number, $N$, of associated sample points (the psips or walkers) is selected in the 9dimensional body-fixed internal configuration space of the three electrons, having a density proportional to the wave function. All $N$ walkers change position in configuration space according to a Monte Carlo procedure based on the property of the corresponding Green's function to reproduce the electronic wave function by an integration over configuration space. ${ }^{26}$ Local energies and their average and standard deviation are calculated by a Monte Carlo importance sampling method after each $\mathrm{N}$-point iteration or each block of several 
such iterations. For each nuclear geometry, the iterative procedure is interrupted after the accumulated standard deviation or a preselected computation time is reached, whichever comes first. In the latter case, the same geometry is used in a subsequent run, until the desired accuracy is obtained.

The computation time increases both with $N$ and with the desired accuracy, and for fixed values of these two parameters it increases with the energy of the configuration. For these reasons, $N$ was chosen by trial and error so as to minimize the computation time for clusters of nuclear geometries with similar energies, and varied from 80000 to 600000 , being mostly about 200000 . The start-up trial configuration was the one used previously, as were the remaining numerical parameters. ${ }^{25}$ The density of grid points in nuclear configuration space was quite high (see Section 3) due to the requirement that the PES fitting accuracy also be high. As a result, the final electronic wave function for a given nuclear geometry provided an excellent trial function for the next neighboring geometry, and resulted in a significant speed-up of the calculation.

The IBM RISC 6000 code used previously ${ }^{26}$ was first ported to a CrayY-MP and carefully vectorized. It was then ported to the Caltech 512 node Intel Paragon, and the original random number generator replaced by an Intel optimized routine. The combination of these two changes increased the speed of the code by a factor of about two. The memory of the Paragon nodes (32 Mbytes or more) was large enough for each nuclear geometry calculation to be done on a single node, and no parallelization was required. To achieve the desired accuracies, it was necessary to run these nodes using 64 bit arithmetic, for which the speed of the present calculations was 42 Mflop per node. The CPU time per geometry on a single node varied from 2.5 to $38 \mathrm{~h}$, depending on the energy and the desired accuracy, with an average of about $5 \mathrm{~h}$. The calculations reported in the present paper included about 76000 geometries, and the total CPU time was about 400000 node $\times$ hours.

\section{Design of the nuclear geometry grid and description of the fitting procedure}

As mentioned in the Introduction, the objective of the present EQMC calculation for $\mathrm{H}_{3}$ was to yield a fitted PES with an absolute accuracy of about $\pm 0.015 \mathrm{kcal} \mathrm{mol}^{-1}$, appropriate for scattering calculations at resonance energies within experimental accuracy. Achievement of such an accuracy requires that two conditions be fulfilled:

(1) The ab initio statistical accuracy of the calculation for each nuclear geometry in the dynamically accessible region should be somewhat higher than the desired fitted one, as fitting procedures of PES surfaces are notoriously difficult and human labor-intensive, leading usually to errors in excess of that of the initial $a b$ initio calculation. A standard deviation of $\pm 0.01 \mathrm{kcal} \mathrm{mol}^{-1}$ for energies of $3 \mathrm{eV}$ and below should be appropriate.

(2) The grid of nuclear geometries should be sufficiently dense so that the energy difference between neighboring points on that grid should be small enough for a simple interpolation scheme, such as a three-dimensional cubic spline, to yield the desired fitting accuracy of about $\pm 0.015 \mathrm{kcal} \mathrm{mol}^{-1}$ in the dynamically accessible region, as well as a maximum error not much larger than that. More efficient global fitting procedures are available, ${ }^{27-30}$ but the present approach guarantees that a relatively simple fitting code can be made to fulfill this quite demanding accuracy requirement without excessive development time. A high density grid of this kind can be achieved if the spacing in each of the three cubic spline directions is chosen to be inversely proportional to the corresponding local partial derivatives. Such a procedure leads, however, to a very irregular grid, so that an intermediate approach involving this criterion in average, together with an approximately regular grid criterion, was adopted and led to the grid parameters described below.

Since the calculated PES surface is to be used in scattering calculations based on the kinematic-rotation-invariant hyperspherical coordinates $\rho, \theta, \phi^{4,6,31,32}$ the nuclear geometry grid was chosen in the internal configuration space for which these variables are polar coordinates. With $\mathrm{A}, \mathrm{B}$ and $\mathrm{C}$ denoting the three hydrogen nuclei, let $\boldsymbol{r}_{1}^{\prime}$ be the Jacobi vector from B to C and $\boldsymbol{r}_{2}^{\prime}$ the one from the center of mass of BC to A and let $\gamma$ be the angle in the 0 to $\pi$ range between these two vectors. The corresponding mass-scaled vectors $\boldsymbol{r}_{1}, \boldsymbol{r}_{2}$ are defined by ${ }^{33}$

$$
\begin{aligned}
& \boldsymbol{r}_{1}=(3 / 4)^{1 / 4} \boldsymbol{r}_{1}^{\prime} \\
& \boldsymbol{r}_{2}=(4 / 3)^{1 / 4} \boldsymbol{r}_{2}^{\prime}
\end{aligned}
$$

The hyperspherical coordinates being considered are given in terms of $r_{1}, r_{2}$ and $\gamma$ by

$$
\begin{aligned}
\rho & =\left(r_{2}^{2}+r_{1}^{2}\right)^{1 / 2} \\
\cos \vartheta & =\left(2 / \rho^{2}\right) r_{2} r_{1} \sin \gamma \quad 0 \leqslant \vartheta \leqslant \pi / 2 \\
\sin \vartheta \cos \phi & =\left(1 / \rho^{2}\right)\left(r_{2}^{2}-r_{1}^{2}\right) \\
\sin \vartheta \sin \phi & =\left(2 / \rho^{2}\right) r_{2} r_{1} \cos \gamma \quad 0 \leqslant \phi<2 \pi
\end{aligned}
$$

The inverses of eqns. (2) are

$$
\begin{aligned}
r_{2} & =\rho[1+\sin \vartheta \cos \phi) / 2]^{1 / 2} & & r_{2} \geqslant 0 \\
r_{1} & =\rho[(1-\sin \vartheta \cos \phi) / 2]^{1 / 2} & & r_{1} \geqslant 0 \\
\tan \gamma & =1 /(\tan \vartheta \sin \phi) & & 0 \leqslant \gamma \leqslant \pi
\end{aligned}
$$

and permit us to obtain $r_{2}, r_{1}$ and $\gamma$ uniquely once $\rho, \theta$ and $\phi$ are given. The three internuclear distances $R_{1}=R_{\mathrm{BC}}, R_{2}=$ $R_{\mathrm{AC}}$, and $R_{3}=R_{\mathrm{AB}}$ are related to $r_{1}^{\prime}, r_{2}^{\prime}$ and $\gamma$ by

$$
\begin{aligned}
& R_{1}=r_{1}^{\prime} \\
& R_{2}=\left(r_{2}^{\prime 2}+r_{1}^{\prime 2} / 4-r_{2}^{\prime} r_{1}^{\prime} \cos \gamma\right)^{1 / 2} \\
& R_{3}=\left(r_{2}^{\prime 2}+r_{1}^{\prime 2} / 4+r^{\prime 2 \prime} r_{1}^{\prime} \cos \gamma\right)^{1 / 2}
\end{aligned}
$$

Eqns. (1) through (4) establish a one-to-one correspondence between the hyperspherical coordinates $\rho, \theta, \phi$ and the internuclear distances $R_{1}, R_{2}, R_{3}$. Thus, once the PES is calculated over a hyperspherical grid, it can be obtained for an arbitrary nuclear geometry defined by $R_{1}, R_{2}, R_{3}$ by first using these equations to obtain $\rho, \vartheta, \phi$. For reasons of symmetry, even though the hyperangle $\phi$ is defined in the 0 to $2 \pi$ range, it suffices to restrict it to the 0 to $\pi / 3$ range. The hyperspherical grid over which we calculated the PES was:

(1) values of $\rho$, in bohr $\left(a_{0}\right): 2.0$ through 3.0 in steps of 0.2 , 3.2 through 3.6 in steps of $0.05,3.8$ through 4.4 in steps of 0.2 and 4.5 through 12 in steps of 0.5 , for a total of 35 values.

(2) The $\theta$ grid, in the 0 to $\pi / 2$ range, and the $\phi$ grid, in the 0 to $\pi / 3$ range, had $n_{9}$ and $n_{\theta}$ equidistant points depending on $\rho$ (in $a_{0}$ ) as follows

$$
\begin{array}{lllll}
2.0 \leqslant \rho \leqslant 2.8 & n_{\vartheta}=46 & \Delta \vartheta=2^{\circ} & n_{\phi}=61 & \Delta \phi=11^{\circ} \\
3.0 \leqslant \rho \leqslant 4.4 & n_{\vartheta}=61 & \Delta \vartheta=1.5^{\circ} & n_{\phi}=61 & \Delta \phi=1^{\circ} \\
4.5 \leqslant \rho \leqslant 12.0 & n_{\vartheta}=31 & \Delta \vartheta=3^{\circ} & n_{\phi}=31 & \Delta \phi=2^{\circ}
\end{array}
$$

(3) In addition, for $3.2 a_{0} \leqslant \rho \leqslant 3.6 a_{0}$, and geometries not very far from collinear, a finer $\vartheta$ grid and $\phi$ grid were used, the smallest grid point spacings being $0.5^{\circ}$ and $0.25^{\circ}$, respectively.

The total number of points on this grid was 71969 . Accurate $a b$ initio calculations were also performed for an additional 3684 nuclear geometries, chosen randomly and not located on this grid, to serve as an unbiased sample for calculating the accuracy of the fitting procedure. The standard deviations selected for the $a b$ initio calculations are given in Table 1, where $V$ stands for the potential energy. They are upper limits to the deviations actually obtained. These 71969 points were then used to generate the fitted PES by a three- 
Table 1 Standard statistical deviations of $a b$ initio EQMC calculations

\begin{tabular}{ll}
\hline Potential energy range/eV & Standard deviation $/ \mathrm{kcal} \mathrm{mol}^{-1}$ \\
\hline$V \leqslant 3$ & \pm 0.010 \\
$3<V \leqslant 4$ & \pm 0.030 \\
$4<V \leqslant 5$ & \pm 0.050 \\
$V>5$ & \pm 0.50 \\
\hline
\end{tabular}

dimensional cubic spline procedure. ${ }^{34}$ Using the additional 3684 random points mentioned above, the root mean square error and the absolute value of the maximum error of this fitted surface were calculated, and are given in Table 2. As can be seen from these tables, for total energies below $3 \mathrm{eV}$ (appropriate for the calculation of the resonance at about 1.5 $\mathrm{eV}$ mentioned in Section 1) both the ab initio and fitting accuracies are equal to $\pm 0.010 \mathrm{kcal} \mathrm{mol}^{-1}$, which is adequate for accurate scattering calculations to be performed.

In addition to the 71969 points described above, ab initio calculations at 157 more points along the normal mode cuts through the saddle point defined in Section 4.2, in the van der Waals region defined in Section 4.3, and for conical intersection configurations defined in Section 4.4, were performed, because of their importance. These points were not incorporated in the data set used for the three-dimensional cubic spline fit of the PES, but used instead to further verify the accuracy of this fit in those regions. The grand total number of geometries at which EQMC ab initio calculations were made was 75810 .

Atomic units were used in the EQMC $a b$ initio code and the resulting energies were transformed into $\mathrm{eV}$ and $\mathrm{kcal} \mathrm{mol}^{-1}$ with the use of the conversion factors 1 hartree $\left(E_{\mathrm{h}}\right)=27.2144$ $\mathrm{eV}$ and $1 \mathrm{eV}=23.0605 \mathrm{kcal} \mathrm{mol}^{-1}$.

\section{Results}

\subsection{Saddle point properties}

One-dimensional cuts of the EQMC PES are examined in this section. A full two-dimensional cut at a constant hyperradius is considered in Section 4.2 and furnishes additional important information about the PES in the vicinity of its saddle point.

We designate by $R^{\ddagger}$ the nearest-neighbor distance at the saddle point, and by $V^{\neq}$the saddle point energy. Let $\theta$ be the angle between the sides $\mathrm{BA}$ and $\mathrm{BC}$ of the $\mathrm{ABC}$ triangle defined in the paragraph preceding eqn. (1). At the saddle point, $\theta=\pi$ and as a result

$$
V^{\ddagger}=V\left(R_{1}=R^{\ddagger}, R_{2}=R^{\ddagger}, \theta=\pi\right)
$$

where the potential energy $V$ is considered to be a function of $R_{1}, R_{2}$ and $\theta$. The cuts of $V$ we consider are the same as those examined by Truhlar and Horowitz, ${ }^{18}$ and are defined as follows: (a) Symmetric stretch cut. We vary the independent variable $R$ and set $R_{1}=R_{2}=R$ and $\theta=\pi$. The associated symmetric stretch normal mode coordinate $\chi_{\mathrm{s}}$ is defined by

$$
\chi_{\mathrm{s}}=\sqrt{3}\left(R-R^{\ddagger}\right)
$$

(b) Bending cut. We vary the independent variable $\theta$ and set $R_{1}=R_{2}=R^{\ddagger}$. The associated bending normal mode coordinate $\chi_{\mathrm{b}}$ is defined by

$$
\chi_{\mathrm{b}}=\frac{1}{2} R^{\ddagger}(\pi-\theta)
$$

(c) Asymmetric stretch cut. We vary the independent variable $R$ and set $R_{1}=R, R_{2}=2 R^{\ddagger}-R$ and $\theta=\pi$. The associated asymmetric stretch normal mode coordinate $\chi_{\mathrm{a}}$ is defined by

$$
\chi_{\mathrm{a}}=R-R^{\ddagger}
$$

In the vicinity of the saddle point $V$ is given approximately by

$$
V\left(R_{1}, R_{2}, \theta\right) \approx V^{\ddagger}+\frac{1}{2} k_{\mathrm{s}} \chi_{\mathrm{s}}^{2}+\frac{1}{2} k_{\mathrm{b}} \chi_{\mathrm{b}}^{2}+\frac{1}{2} k_{\mathrm{a}} \chi_{\mathrm{a}}^{2}
$$

The zero-point energies, $E_{0}^{(i)}$, associated with these three normal modes are given by

$$
E_{0}^{(i)}=(1 / 2) \hbar\left(k_{i} / m\right)^{1 / 2} \quad i=\mathrm{s}, \mathrm{b}, \mathrm{a}
$$

where $m=(2 / 3) m_{\mathrm{H}}$ and $m_{\mathrm{H}}$ is the mass of the hydrogen atom.

Figs. 1-3 display plots of the normal mode cuts of $V v s$. the corresponding normal mode coordinates, for the fitted EQMC, BKMP2 and LSTH PESs, as well as differences between the last two and the first. The corresponding barrier heights, saddle point configurations and normal mode force constants and energies, obtained from a normal mode analysis of the respective fitted PESs, are given in Table 3. From panel (b) of each of these three figures it can be seen that at the saddle point configuration the difference curves have a slope very close to zero, corresponding to saddle point curvatures in panel (a) which should be nearly equal. As a result, the normal mode force constants of each of the three PESs being considered should be very close to each other. As shown in Table 3 , this indeed is the case. Once points slightly displaced from the saddle point are considered, however, differences between the variations in the PESs of $0.02 \mathrm{kcal} \mathrm{mol}^{-1}$ are rapidly reached. For example, an examination of Fig. 1(b) indicates that as the nearest neighbor symmetric stretch distance is increased from $1.64 a_{0}$ through the saddle point value of 1.757 $a_{0}$ to $1.87 a_{0}$, a change of only $0.23 a_{0}$, the BKMP2 potential increases by $0.066 \mathrm{kcal} \mathrm{mol}^{-1}$ more than the EQMC one. This contrasts with the fact that at the saddle point these two potentials are equal to within $0.01 \mathrm{kcal} \mathrm{mol}^{-1}$ and that their harmonic symmetric stretch zero point energies are equal to within $0.001 \mathrm{kcal} \mathrm{mol}^{-1}$. Therefore, examining the characteristics of the PES at the saddle point only (as given in Table 3), rather than over a more extended neighborhood (spanned by the abcissas of Figs. 1-3) can be misleading. Since, as discussed in Section 1, changes in the PES in the saddle point region of about $0.015 \mathrm{kcal} \mathrm{mol}^{-1}$ are expected to have a detectable effect on resonance properties, it is expected on the basis of the changes along the symmetric stretch motion that these properties for the EQMC and BKMP2 PESs may differ

Table 2 Accuracy of the cubic spline fitted EQMC PES

\begin{tabular}{llll}
\hline Potential energy range/eV & Number of geometries & Root mean square error/kcal mol ${ }^{-1}$ & ${\text { Maximum error } / \mathrm{kcal} \mathrm{mol}^{-1}}$ \\
\hline$V \leqslant 2$ & 19837 & \pm 0.009 & 0.011 \\
$2<V \leqslant 3$ & 28773 & \pm 0.010 & 0.018 \\
$3<V \leq 5$ & 21762 & \pm 0.038 & 0.050 \\
$V>5$ & 5438 & \pm 0.50 & 0.60 \\
\hline
\end{tabular}


Table 3 Saddle point properties of the EQMC, BKMP2 and LSTH PESs

\begin{tabular}{lccc}
\hline Property & EQMC & BKMP2 & LSTH \\
\hline$R^{\ddagger} / a_{0}$ & 1.75702 & 1.7570 & 1.7570 \\
$E^{\ddagger} / \mathrm{kcal} \mathrm{mol}^{-1} a$ & 9.613 & 9.610 & 9.802 \\
$k_{\mathrm{s}} / \mathrm{h} \mathrm{bohr}^{-2}$ & 0.10730 & 0.1073 & 0.1077 \\
$k_{\mathrm{b}} / \mathrm{h} \mathrm{bohr}^{-2}$ & 0.02086 & 0.0209 & 0.0210 \\
$k_{\mathrm{a}} / \mathrm{h} \mathrm{bohr}^{-2}$ & -0.05745 & -0.0576 & -0.0577 \\
$E_{0}^{\mathrm{s}} / \mathrm{kcal} \mathrm{mol}^{-1} \quad b$ & 2.9368 & 2.937 & 2.942 \\
$E_{0}^{\mathrm{b}} / \mathrm{kcal} \mathrm{mol}^{-1} b$ & 1.295 & 1.297 & 1.299 \\
$E_{0}^{\mathrm{a}} / \mathrm{kcal} \mathrm{mol}^{-1} \quad b$ & $2.150 i$ & $2.151 i$ & $2.154 i$
\end{tabular}

${ }^{a} E^{\ddagger}=V^{\ddagger}-V_{\mathrm{e}}$ where $V_{\mathrm{e}}$ is the potential energy of $\mathrm{H}_{2}$ at its equilibrium internuclear distance. ${ }^{35} b$ The value of $m_{\mathrm{H}}$ used to compute the harmonic zero point energies was $1837.15 \mathrm{~m}_{\mathrm{e}}$.

non-negligibly. Similarly, an analysis of Fig. 3(b) shows that along the antisymmetric stretch, as $R_{1}$ increases from its saddle point value to $0.37 a_{0}$ above it, the BKMP2 energy decreases by about $0.11 \mathrm{kcal} \mathrm{mol}^{-1}$ more than the EQMC one, another non-negligible difference. The effect of bending the saddle point configuration is less pronounced: Fig. 2(b) indicates that a bend of about $17^{\circ}$ away from collinearity increases the BKMP2 potential by only about $0.01 \mathrm{kcal}$ $\mathrm{mol}^{-1}$ more than the EQMC one. A similar analysis of the differences between the LSTH and EQMC curves of Figs. 1-3 and of the corresponding parameters in Table 3 shows that not only do the corresponding barrier heights differ by the relatively large amount of $0.19 \mathrm{kcal} \mathrm{mol}^{-1}$, but that in addi-
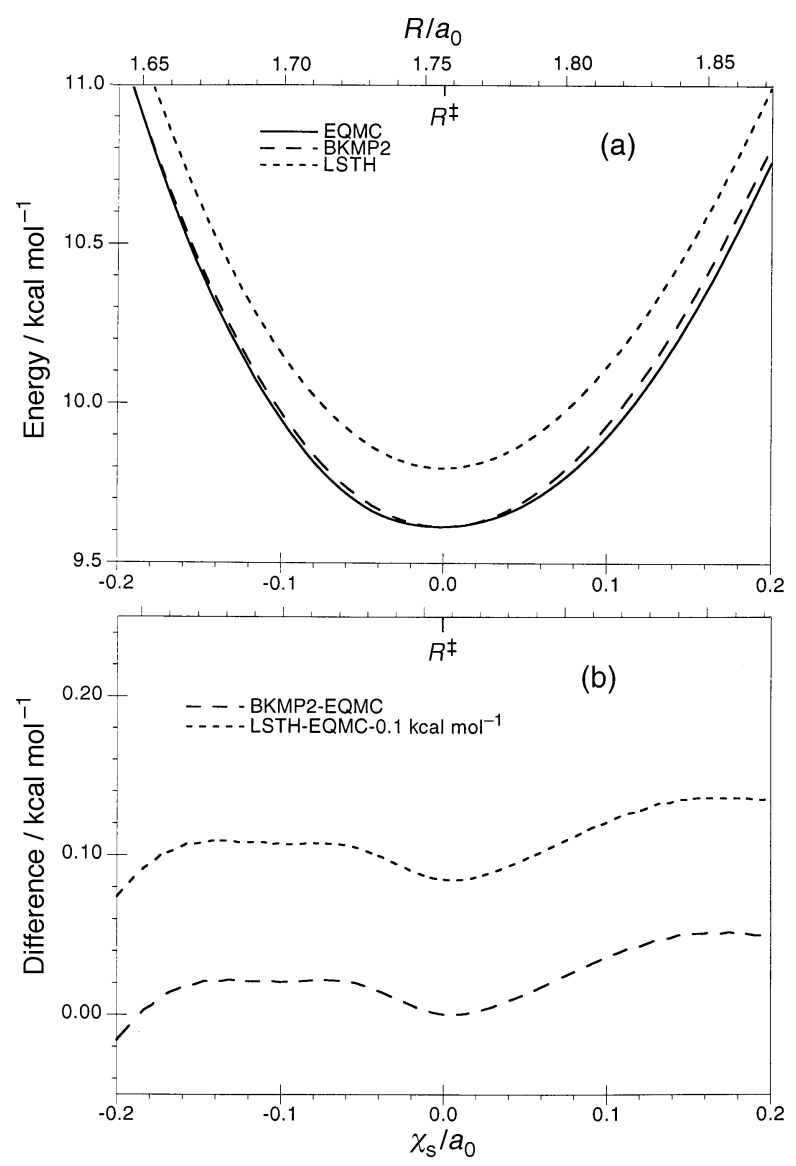

Fig. 1 Fitted PES energies and their differences along the symmetric stretch normal mode cut through the saddle point. Full line, EQMC potential; --- BKMP2 potential; ---- LSTH potential. The lower abcissa is the symmetric stretch normal mode coordinate, and the upper abcissa the corresponding nearest neighbor interatomic distance. $R^{\ddagger}$ depicts the value of this distance at the saddle point. (a) The potential energies. (b) The difference between the BKMP2 (---) or the LSTH (---) potential energy and the present EQMC one. $0.1 \mathrm{kcal}$ $\mathrm{mol}^{-1}$ was subtracted from the latter difference before plotting.
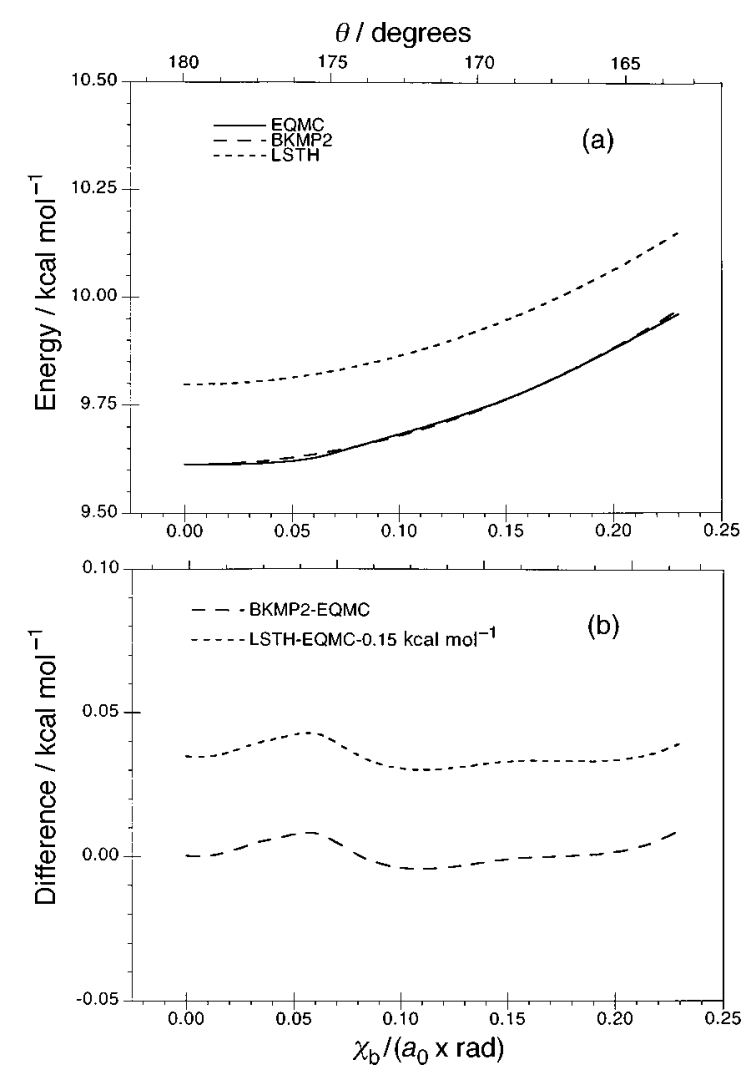

Fig. 2 Same as for Fig. 1, but for the bend normal mode. The upper abcissa is the bending angle. In panel (b), $0.15 \mathrm{kcal} \mathrm{mol}^{-1}$ was subtracted from the difference between the LSTH and EQMC potentials before plotting.

tion the shapes of these two PESs (in the neighborhood of the saddle spanned by the abcissas of those figures) are more different than are the shapes of the BKMP2 and EQMC PESs. A conclusion of this analysis is that the differences between these three PESs should be examined in greater detail in the extended neighborhood of the saddle point rather than along the saddle point normal mode displacement directions only. This is done in Section 4.2.

Fig. 4 displays the energy along the minimum energy paths (MEPs) for the fitted EQMC, BKMP2 and LSTH PESs and the differences between the corresponding MEP energies. The MEPs were calculated from a collinear cut $(\theta=0)$ of the fitted PESs in the two-dimensional cartesian space defined by $R_{1}$ and $R_{2}$. In that figure, the abcissa $s$ is the signed distance along the MEP with $s=0$ corresponding to the saddle point. It can be seen from panel (b) that the difference between the BKMP2 and EQMC MEP energies is in the range $-0.05 \mathrm{kcal}$ $\mathrm{mol}^{-1}$ to $0.12 \mathrm{kcal} \mathrm{mol}^{-1}$, leading again to the expectation that significant differences will be found between the resonance energies calculated using these two PESs. The difference between the LSTH and EQMC MEP energies is even more pronounced, ranging from $-0.11 \mathrm{kcal} \mathrm{mol}^{-1}$ to $0.19 \mathrm{kcal}$ $\mathrm{mol}^{-1}$.

\subsection{Equipotential contours at constant hyperradii}

A convenient way for displaying the three-dimensional potential energy surface $V(\rho, \vartheta, \phi)$ is to plot equipotential contours of $V$ at constant values of the hyperradii as two-dimensional curves. To that effect we define a system of circular polar coordinates for which the radius is $\rho \vartheta$ and the polar angle, measured counter-clockwise from a positive vertical axis, is $\phi$. This is done in panels (a), (b) and (c) of Fig. 5 for the fitted EQMC PES, for the representative hyperradii $\rho=2.0 a_{0}, 3.27$ $a_{0}$ and $6.0 a_{0}$. The first of these three values represents a hyperradius which is classically inaccessible at $E=2.5 \mathrm{eV}$ and 


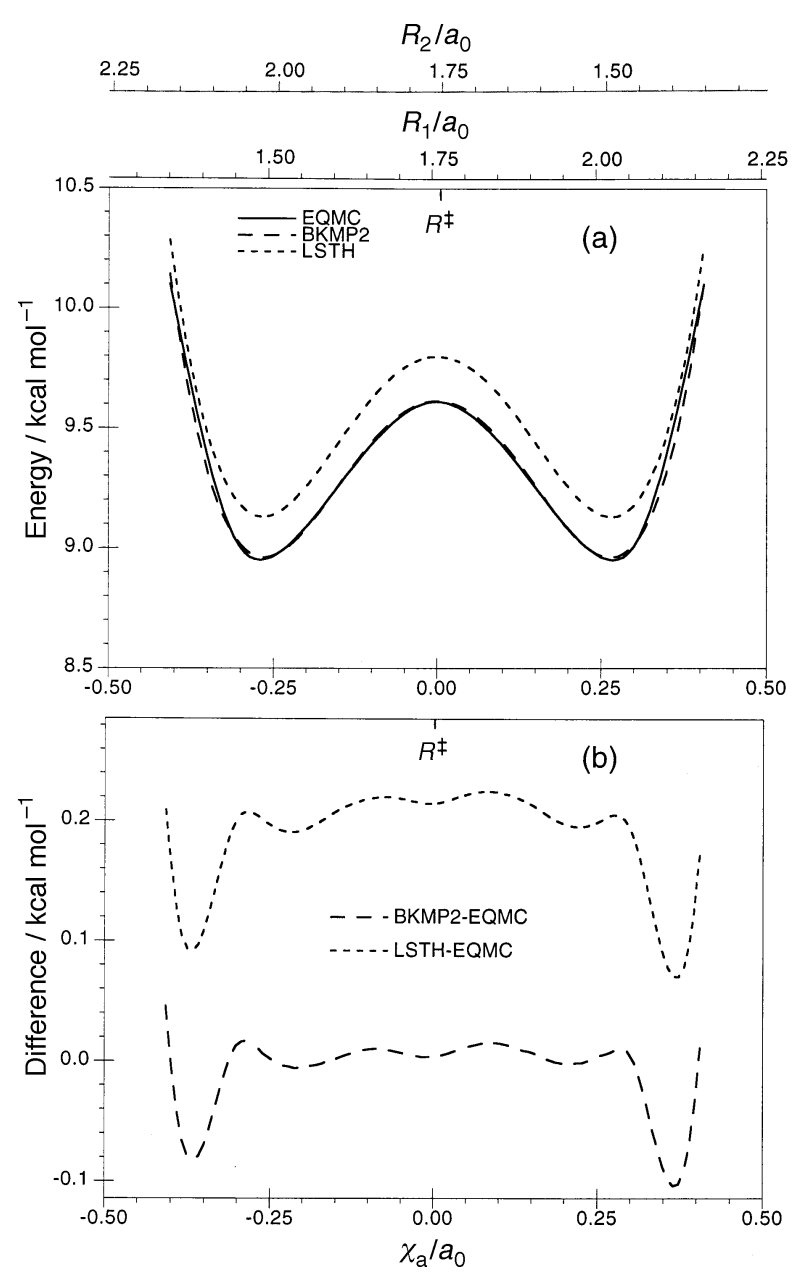

Fig. 3 Same as for Fig. 1, but for the antisymmetric normal mode. The upper abcissas are: (1) $R_{1}$, one of the two nearest neighbor distances; (2) $R_{2}$, the other nearest neighbor distance. The sum of the two is constant and equals twice the corresponding saddle point distance.

below (since the lowest energy equipotential exceeds $2.5 \mathrm{eV}$ ), the second one corresponds to the hyperradius passing through the three equivalent saddle points designated by the crosses on periphery of the full circle, and the third one is a hyperradius for which the three arrangement channel regions are separated by a barrier in excess of $3.0 \mathrm{eV}$. The shaded regions in panels (b) and (c) between the $2.0 \mathrm{eV}$ equipotentials represent the classical energetically accessible regions of the corresponding hyperspheres at $E=2.0 \mathrm{eV}$. Scattering wave functions at this energy and below will be concentrated predominantly in these regions.

The corresponding figures for the BKMP2 and LSTH PESs are analogous to the EQMC one, and on the scale used in Fig. 5 the contours for these surfaces would be practically indistinguishable from one another. For this reason, in panels (d), (e) and (f) contours of the difference between the BKMP2 and EQMC PESs are displayed, and in (g), (h) and (i) those for the difference between the LSTH and EQMC PESs are represented. In panel (e) the shaded area between the two contours labeled $0.02 \mathrm{kcal} \mathrm{mol}^{-1}$ in the center of the figure and the area between the contours corresponding to $0.02 \mathrm{kcal}$ $\mathrm{mol}^{-1}$ and $-0.02 \mathrm{kcal} \mathrm{mol}^{-1}$ (dashed) in the periphery of the figure represent regions for which the differences between the BKMP2 and EQMC PESs are smaller in absolute values than $0.02 \mathrm{kcal} \mathrm{mol}^{-1}$. Comparison between these regions and the classically accessible region of panel (b) at $2.0 \mathrm{eV}$ indicates that for a large portion of that region the absolute value of the difference between the BKMP2 and EQMC PESs exceeds $0.02 \mathrm{kcal} \mathrm{mol}^{-1}$ and could lead to non-negligible differences
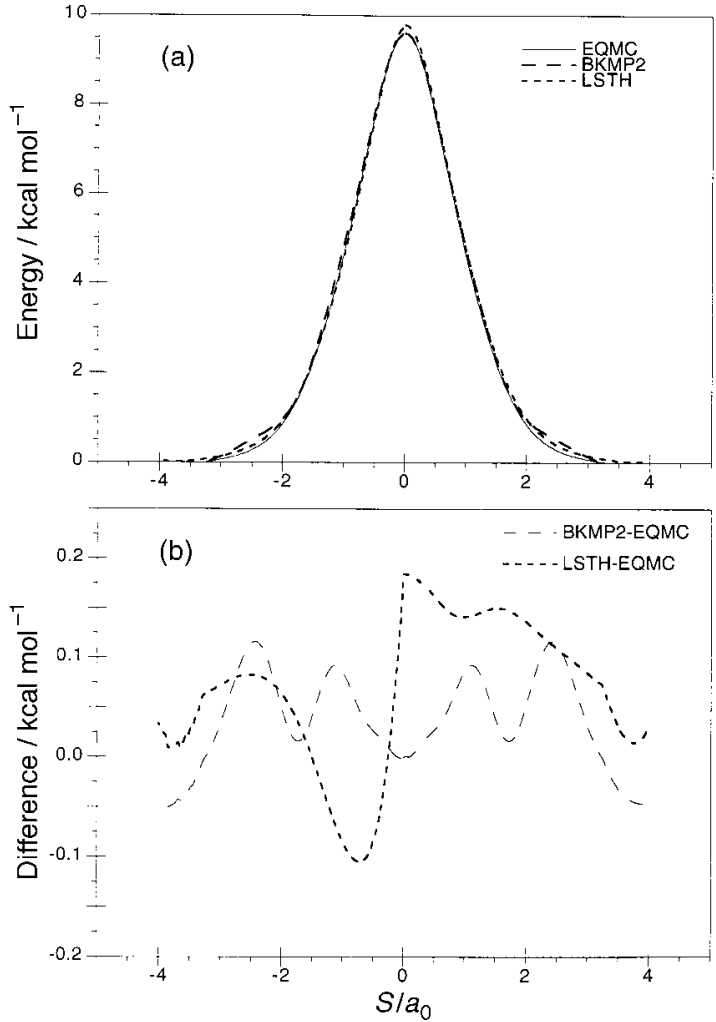

Fig. 4 Minimum energy path potentials and their differences, for the EQMC, BKMP2 and LSTH fitted PESs, as a function of the distance $s$ along the MEP. (a) The MEP energies. (b) The BKMP2 - EQMC and LSTH - EQMC differences.

between the properties of the resonances obtained using these two surfaces. At $\rho=6 a_{0}$, the shaded areas in panels (c) and (f) have the same meaning as for panels (b) and (c) and the effect just discussed is similar but less pronounced than at 3.27 $a_{0}$. Analogous observations are valid for the differences between the LSTH and EQMC PESs displayed in panels (h) and (i), these differences being, however, more pronounced than between the BMKP2 and EQMC surfaces. As a result, the shaded areas in these panels are imperceptible. This analysis reinforces the prediction arrived at in Section 4.1.

\subsection{The van der Waals region}

$A b$ initio EQMC calculations were also performed in the important van der Waals region ${ }^{36}$ with a lower selected statistical error of $\pm 0.005 \mathrm{kcal} \mathrm{mol}^{-1}$, in order to better determine the characteristics of this well, which has a depth of the order of $0.05 \mathrm{kcal} \mathrm{mol}^{-1}$. Fig. 6 displays the EQMC, BKMP2 and LSTH fitted potentials for collinear configurations for which the diatomic internuclear distance was fixed at 1.449 $a_{0}$, as a function of the distance from the third atom to the center-of-mass of the diatom. As can be seen, the absolute value of the difference between the first two potentials never exceeds $0.005 \mathrm{kcal} \mathrm{mol}^{-1}$, indicating that they are equal to each other within the EQMC accuracy. The difference between the LSTH and EQMC van der Waals wells is, as expected, significantly larger.

\subsection{Configurations at and near the conical intersection}

The fitted energies for equilateral triangle configurations are displayed in Fig. 7 as a function of the length of the side of the triangle for the three PESs being considered, as are their differences. The EQMC curve displays a minimum of $2.7207 \mathrm{eV}$, which represents the minimum conical intersection energy. For the BKMP2 and LSTH PESs this value is $2.725 \mathrm{eV}^{20}$ and $2.756 \mathrm{eV}$, respectively. The value of $r$ corresponding to 

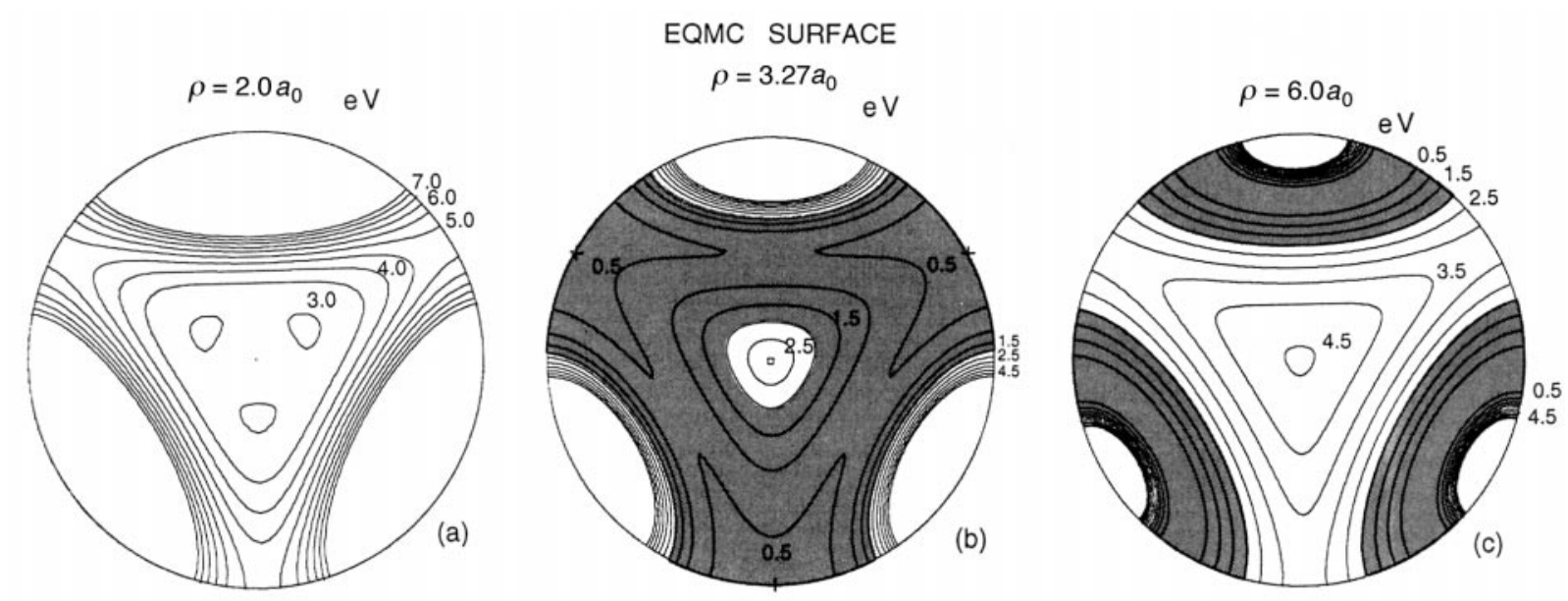

BKMP2-EQMC SURFACE
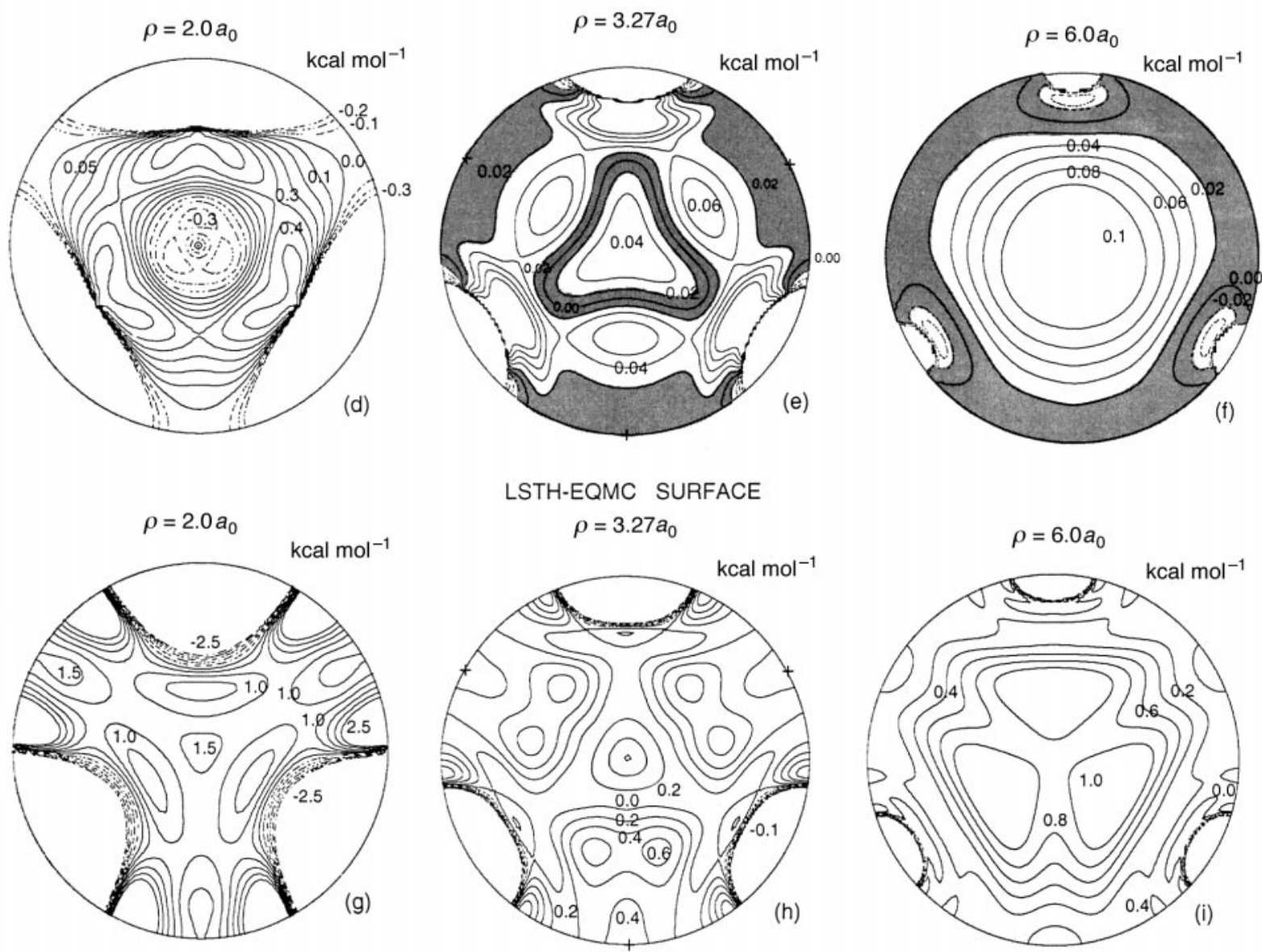

Fig. 5 Equipotential contours of the fitted PES energies and their difference, at constant hyperradii $\rho$. The points in the plots have planar polar coordinates $\rho \vartheta$ (with $\vartheta$ in radians), the distance to the center of the circles depicted, and polar angle $\phi$ with respect to a vertical axis whose origin is at those centers and pointing upwards. The radii of those circles are $\rho \pi / 2$, and determine the distance scale of the plots. The periphery of the circles depict collinear configurations, whereas their centers correspond to equilateral configurations. (a), (b) and (c), Equipotentials of the fitted EQMC PES, in eV, for $\rho=2.0 a_{0}, 3.27 a_{0}$ (the saddle point hyperradius) and $6.0 a_{0}$, respectively. The three crosses on the periphery of the circle in (b) locate the three equivalent saddle points. (d), (e) and (f): equipotentials of the difference between the BKMP2 and EQMC fitted PESs, in $\mathrm{kcal} \mathrm{mol}^{-1}$, at the indicated values of $\rho$. (g), (h) and (i), The same as (c), (d) and (e) with the BKMP2 PES replaced by the LSTH one. See text for the meaning of the shaded areas in panels (b), (c), (e), and (f).

these minima is the same for all three PESs, $1.971 a_{0}$. The importance of the first excited state of $\mathrm{H}_{3}$ for reactive scattering calculations should set in at almost the same energy for the EQMC and BMKP2 PESs and at a slightly higher energy for the LSTH, but this small difference should have a negligibly small effect on the corresponding one- or even twoelectronic state cross-sections.

In Fig. 8, equipotential contours of the fitted EQMC PES, as well as of the differences between the BKMP2 or LSTH
PES and the EQMC energies, are displayed in the near neighborhood of the conical intersection configuration at the saddle point hyperradius. The coordinates of this figures are the same as those of Fig. 5 and the curves are an enlarged scale plot of the central regions of panels (b), (e) and (h) of that figure. It can be seen that close to the conical intersection, the BKMP2 PES is about $0.05 \mathrm{kcal} \mathrm{mol}^{-1}$ or more higher than the EQMC one, whereas for the LSTH PES this difference is $0.2 \mathrm{kcal}$ $\mathrm{mol}^{-1}$ or more. Since this region of the PESs is not expected 

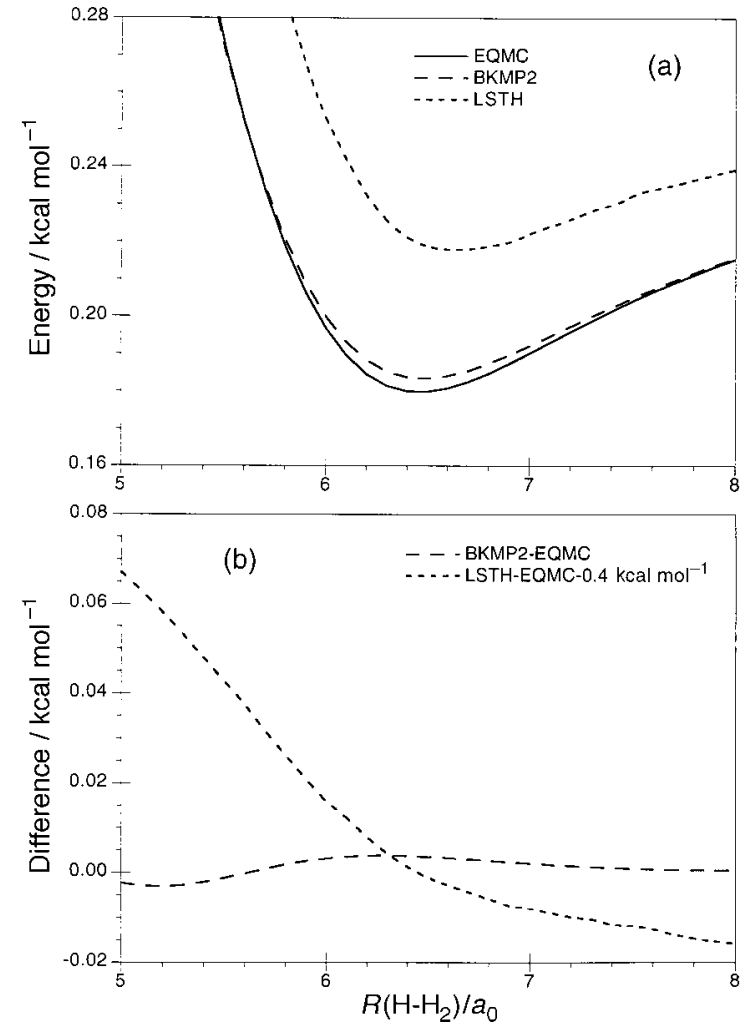

Fig. 6 Potential energies and their differences in the van der Waals region. The configurations are collinear, with the diatomic distance $r$ maintained constant at $1.449 a_{0}$. The abcissa $R\left(\mathrm{H}-\mathrm{H}_{2}\right)$ is the distance in $a_{0}$ of the third atom to the center-of-mass of the diatom. (a) The fitted EQMC (full line), BKMP2(---) and LSTH (----) energies, in $\mathrm{kcal} \mathrm{mol}^{-1}$. (b) The difference between the fitted BKMP2 and EQMC energies (---) and between the LSTH and EQMC energies (----), with $0.4 \mathrm{kcal} \mathrm{mol}^{-1}$ having been subtracted from the latter before plotting.

to affect cross-sections at an energy of $1.6 \mathrm{eV}$ or lower, these differences should have a negligible effect on the resonance energy in the neighborhood of $1.5 \mathrm{eV}$.

The fitted EQMC PES will be made available upon request.

\section{Discussion}

\subsection{Ab initio and fitting accuracies}

The $a b$ initio and fitting accuracies reported in Tables 1 and 2 are of unprecedented quality for the $\mathrm{H}_{3}$ system, the fitted PES being an order of magnitude better than those for any previous PES for this system. For energies below $3 \mathrm{eV}$ both the $a b$ initio and fitting accuracies are $\pm 0.010 \mathrm{kcal} \mathrm{mol}^{-1}$, and the absolute value of the maximum fitting error is $0.018 \mathrm{kcal}$ $\mathrm{mol}^{-1}$. By comparison, for the PES of the next highest quality, the BKMP2 one, ${ }^{20}$ for "non-compact" configurations, the ab initio accuracy is $0.06 \mathrm{kcal} \mathrm{mol}^{-1}$ (the estimated uncertainty in the extrapolation plus full CI correction), the fitting accuracy is $\pm 0.11 \mathrm{kcal} \mathrm{mol}^{-1}$, and the absolute value of the maximum fitting error is $1.1 \mathrm{kcal} \mathrm{mol}^{-1}$. As discussed in Section 1, a $\pm 0.015 \mathrm{kcal} \mathrm{mol}^{-1}$ fitted PES accuracy is required for an accurate prediction of resonances in this system.

\subsection{Computational effort}

As mentioned at the end of Section 2, the total CPU time used in the calculation of the present EQMC PES was about 400000 node $\times$ hours of a 512 node Intel Paragon distributed memory computer, corresponding to about $800 \mathrm{~h}$ of the full machine. This is an early commercial massively parallel computer, having a sustained speed for the present calculations of
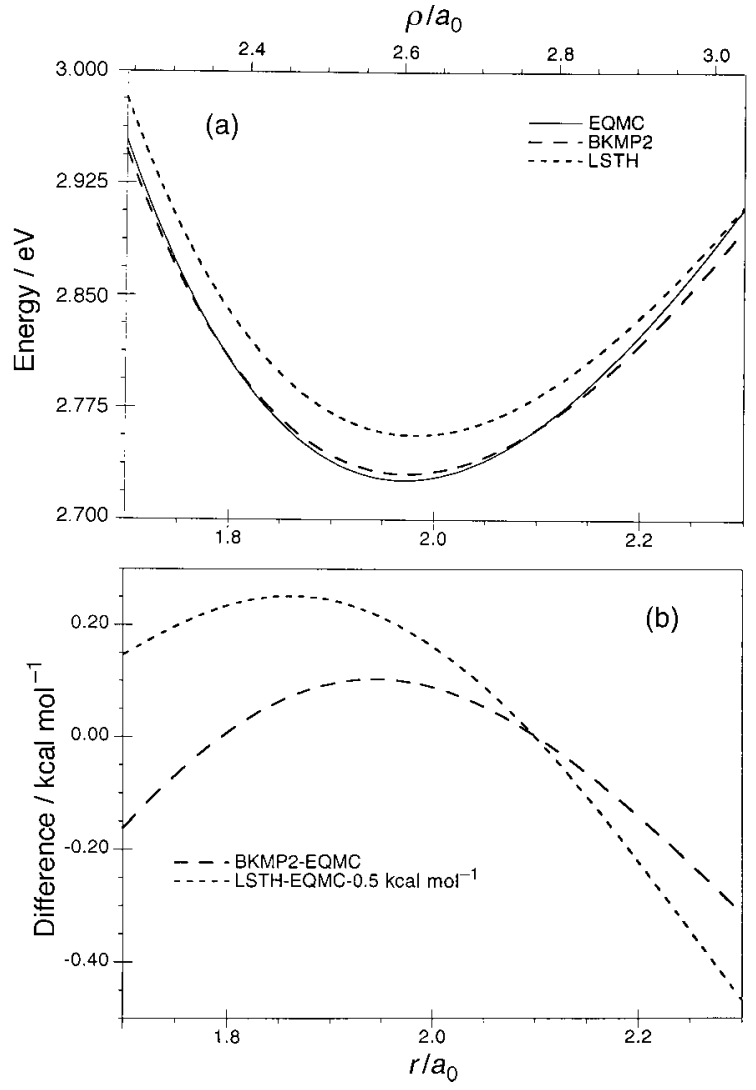

Fig. 7 Potential energies and their differences for equilateral triangle configurations, as a function of the length $r$ of the side of the triangle (lower abcissa) and the corresponding hyperradius $\rho$ (upper abcissa), in $a_{0}$. (a) The fitted EQMC (full line), BKMP2(---) and LSTH (----) energies in eV. (b) The difference between the fitted BKMP2 and EQMC energies (---) and LSTH and EQMC energies (----) in kcal $\mathrm{mol}^{-1}$, with $0.5 \mathrm{kcal} \mathrm{mol}^{-1}$ having been subtracted from the latter before plotting.

42 Mflops per node or about 21 Gflops for the whole machine. Current state-of-the-art parallel computers, such as the ASCI Red at Sandria National Laboratories in Albuquerque, NM, have sustained speeds for the present kind of calculations 66 times greater, ${ }^{37}$ permitting this calculation to be performed in about $12 \mathrm{~h}$. As the speed of parallel computers continues to grow rapidly, and as they become more generally available, this amount of CPU time should not be considered excessive, given the unprecedented level of accuracy attained for this $\mathrm{H}_{3}$ system.

\subsection{Zero total angular momentum resonance energy}

Using the fitted EQMC PES described in this paper, preliminary zero total angular momentum calculations were performed for the $\mathrm{H}+\mathrm{D}_{2} \quad(v=0, j=0) \rightarrow \mathrm{HD} \quad\left(v^{\prime}=0\right.$, $\left.j^{\prime}=5\right)+\mathrm{D}$ reaction. ${ }^{38}$ The resonance energy was found to be $1.419 \mathrm{eV}$, compared to $1.442 \mathrm{eV}$ for the BKMP2 surface and $1.497 \mathrm{eV}$ for the LSTH one. Thus, in spite of the almost equal saddle point properties displayed in Table 3 for the EQMC and BKMP2 PESs, the differences displayed in Fig. 3 and discussed in Section 4.2 were enough to shift the EQMC resonance energy downwards $23 \mathrm{meV}\left(0.53 \mathrm{kcal} \mathrm{mol}^{-1}\right)$ with respect to the BKMP2 one. This shift is about one half of the energy width of the BKMP2 differential cross-section resonance. If, after a full scattering calculation is performed, the magnitude of this shift is retained in the EQMC differential cross-section, these new results will be of help in guiding the experimental search for this resonance.

\subsection{Additional corrections to the EQMC PES}

Consider a one-electronically-adiabatic-state approximation, 
(a)

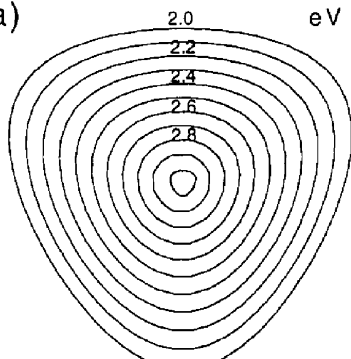

BKMP2-EQMC SURFACE

(b)

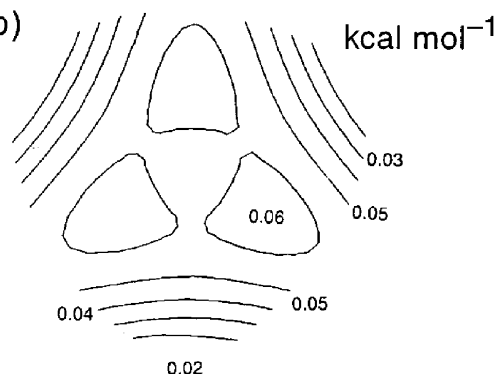

LSTH-EQMC SURFACE

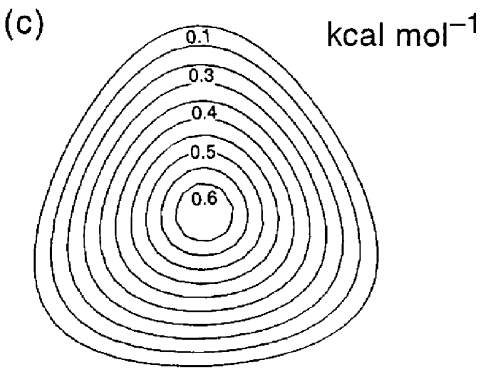

Fig. 8 Contours of the EQMC PES and of the differences between the BKMP2, LSTH and EQMC PESs in the neighborhood of the conical intersection. These are enlarged versions of the central regions of Fig. 5, panels (b), (e) and (h), corresponding to the saddle point hyperradius of $3.27 a_{0}$.

as is appropriate when the energy of the ground electronic state is sufficiently lower than that of the first excited state, and the coupling between these two states is small. This is the case for $\mathrm{H}_{3}$ at energies of the order of $2 \mathrm{eV}$ or lower. In this case, for a real electronic wave function, the nuclear motion Schrödinger equation has a zero first derivative diagonal coupling element, but the second derivative one, which is the expectation value of the nuclear kinetic energy operator with respect to the ground electronic state wave function, does not vanish. This last element is a potential energy term that must be added to the usual potential energy function. ${ }^{39}$ It can be evaluated by a simple expression justified by Handy and Lee $^{40}$ and proven rigorously by Kutzelnigg. ${ }^{41}$ This term has been calculated for the ground electronic state of $\mathrm{H}_{3}{ }^{+42}$ for which it varies from about $12 \mathrm{meV}$ to about $17 \mathrm{meV}(\sim 0.28$ $\mathrm{kcal} \mathrm{mol}{ }^{-1}$ to $0.39 \mathrm{kcal} \mathrm{mol}^{-1}$ ) as the nuclear geometry is scanned, a relatively small variation. It should be noted parenthetically that for the $\mathrm{H}_{3}{ }^{+}$system, the accuracy obtained in the calculation ${ }^{42}$ and fitting ${ }^{43}$ of the PES was better than $0.012 \mathrm{meV}\left(0.00028 \mathrm{kcal} \mathrm{mol}^{-1}\right)$. This extraordinary accuracy, almost two orders of magnitude higher than that of the present $\mathrm{H}_{3}$ EQMC PES, is partly due to the explicit inclusion of the interelectronic distance in the basis functions used in the variational $a b$ initio calculation for $\mathrm{H}_{3}{ }^{+}$. For the $\mathrm{H}_{3}$ system, the lack of a deep well in the PES implies that the magnitude of the second derivative coupling element should be smaller than for $\mathrm{H}_{3}{ }^{+}$and that it should vary less over the range of important geometries. As a result, it should suffice to calculate it over a sparse nuclear geometry grid, of the order of a few hundred points.

A second correction to be considered is the relativistic correction. It has been calculated for $\mathrm{H}_{2},{ }^{44}$ and for energies up to $3 \mathrm{eV}$ (with respect to the bottom of the diatomic well) it is in the range of from $-0.0058 \mathrm{kcal} \mathrm{mol}{ }^{-1}$ to $-0.0112 \mathrm{kcal}$ $\mathrm{mol}^{-1}$. For an isolated $\mathrm{H}$ atom this correction is -0.00417 kcal $\mathrm{mol}^{-1}$. As a result, for the system $\mathrm{H}_{2}+\mathrm{H}$, with the $\mathrm{H}$ atom far from the $\mathrm{H}_{2}$ molecule, the relativistic correction for energies below $3 \mathrm{eV}$ is in the range of $-0.0100 \mathrm{kcal} \mathrm{mol}^{-1}$ to $-0.0154 \mathrm{kcal} \mathrm{mol}^{-1}$. For the saddle point configuration of $\mathrm{H}_{3}$, a rough estimate of this correction is $3 / 2$ of $-0.0061 \mathrm{kcal}$ $\mathrm{mol}^{-1}$ (the value for $\mathrm{H}_{2}$ at an internuclear distance of 1.757 $a_{0}$ ), namely $-0.0091 \mathrm{kcal} \mathrm{mol}^{-1}$. From these considerations, we estimate that for the $\mathrm{H}_{3}$ system at energies below $3 \mathrm{eV}$, the relativistic correction should be in the range from about $-0.010 \mathrm{kcal} \mathrm{mol}^{-1}$ to about $-0.016 \mathrm{kcal} \mathrm{mol}^{-1}$, and therefore should have a negligible effect on the scattering crosssections, even for resonance energies. As a result, this correction does not have to be included in such calculations.

\section{Summary}

An EQMC PES for $\mathrm{H}_{3}$ has been calculated having ab initio and fitting accuracies of $\pm 0.01 \mathrm{kcal} \mathrm{mol}^{-1}$ for energies smaller than $3 \mathrm{eV}$. This is an order of magnitude more accurate than the best previously available PES for this system. After a second derivative diagonal coupling element of the order of $0.3 \mathrm{kcal} \mathrm{mol}^{-1}$ or less is calculated for a sparse nuclear grid and added to it, this PES should be able to predict resonance energies for this system and its isotopomers to an absolute accuracy of about $0.6 \mathrm{meV}\left(0.014 \mathrm{kcal} \mathrm{mol}^{-1}\right.$ or $5 \mathrm{~cm}^{-1}$ ), and relativistic corrections should have a very minor effect on this prediction. This accuracy should be adequate to give reliable guidance to the experimental search of these resonances.

\section{Acknowledgements}

Financial support from NSF Grants No. CHE 9632816 and CHE 9810050 is gratefully acknowledged. The calculations reported were performed on the 512 node Intel Paragon at Caltech's Center for Advanced Computing Research. We thank the staff of the Center for their help. We also thank Dr. Drake L. Diedrich for calculating some points used for an independent verification of the statistical error in the present calculations.

\section{References}

1 J. Z. H. Zhang and W. H. Miller, Chem. Phys. Lett., 1988, 153, 465

2 M. Mladenovic, M. Zhao, D. G. Truhlar, D. W. Schwenke and D. J. Kouri, J. Phys. Chem., 1988, 92, 7035 .

3 D. E. Manolopoulos and R. E. Wyatt, Chem. Phys. Lett., 1989, 159, 123.

4 J. M. Launay and M. Le Dourneuf, Chem. Phys. Lett., 1989, 163, 178

5 M. Zhao, D. G. Truhlar, D. W. Schwenke and D. J. Kouri, J Phys. Chem., 1990, 94, 7074.

6 Y-S. M. Wu, A. Kuppermann and B. Lepetit, Chem. Phys. Lett., 1991, 186, 319 .

7 W. H. Miller and J. Z. H. Zhang, J. Phys. Chem., 1991, 95, 12.

8 T. J. Park and J. C. Light, J. Chem. Phys., 1991, 96, 8853.

9 D. Neuhauser, R. S. Judson, D. J. Kouri, D. E. Adelman, N. E. Shafer, D. A. V. Kliner and R. N. Zare, Science, 1992, 257, 519.

10 S. L. Mielke, R. S. Friedman, D. G. Truhlar and D. W. Schwenke, Chem. Phys. Lett., 1992, 118, 359.

11 Y-S. M. Wu and A. Kuppermann, Chem. Phys. Lett., 1993, 201, 178.

12 A. Kuppermann and Y-S. M. Wu, Chem. Phys. Lett., 1993, 205, 577; erratum, 1993, 213, 636.

13 S. L. Mielke, D. G. Truhlar and D. W. Schwenke, J. Phys. Chem., 1994, 98, 1053

14 A. Kuppermann and Y-S. M. Wu, Chem. Phys. Lett., 1995, 241, 229; erratum, 1995, 243, 586.

15 A. Kuppermann, in Dynamics of Molecules and Chemical Reactions, ed. R. E. Wyatt and J. Z. H. Zhang, Marcel Dekker, New York, 1996, pp. 411-472.

16 D. Neuhauser, R. S. Judson, M. Baer and D. J. Kouri, J. Chem. Phys., 1997, 93, 727. 
17 P. Siegbahn and B. Liu, J. Chem. Phys., 1978, 68, 2457.

18 D. G. Truhlar and C. J. Horowitz, J. Chem. Phys., 1978, 68, 2466; erratum, $1979, \mathbf{7 1}, 1514$

19 A. J. C. Varandas, F. B. Brown, C . A. Mead, D. G. Truhlar and N. Blais, J. Chem. Phys., 1987, 86, 6258.

20 A. I. Boothroyd, W. J. Keogh, P. G. Martin and M. R. Peterson, J. Chem. Phys., 1996, 104, 7139.

21 J. B. Anderson, C. A. Traynor and B. M. Boghosian, J. Chem. Phys., 1991, 95, 7418.

22 J. B. Anderson, J. Chem. Phys., 1992, 96, 3702.

23 D. L. Diedrich and J. B. Anderson, Science, 1992, 258, 786.

24 J. B. Anderson, C. A. Traynor and B. M. Boghosian, J. Chem. Phys., 1993, 99, 345.

25 D. L. Diedrich and J. B. Anderson, J. Chem. Phys., 1994, 100, 8089.

26 J. B. Anderson, in Quantum Mechanical Electronic Structure Calculations with Chemical Accuracy, ed. S. R. Langhoff, Kluwer, Dordrecht, 1995, pp. 1-45.

27 D. K. Hoffman, A. Frishman and D. J. Kouri, Chem. Phys. Lett., 1996, 262, 393.

28 A. Frishman, D. K. Hoffman and D. J. Kouri, J. Chem. Phys., 1997, 107, 804

29 T. Hollebeck, T-S. Ho and H. Rabitz, J. Chem. Phys., 1997, 106, 7223.

30 G. C. Schatz, A. Papaioannou, L. A. Pederson, L. B. Harding, T. Hollebeck, T-S. Ho and H. Rabitz, J. Chem. Phys., 1997, 107, 2340 .
31 B. R. Johnson, J. Chem. Phys., 1980, 73, 5051

32 A. Kuppermann, in Advances in Molecular Vibrations and Collision Dynamics, ed. J. Bowman, JAI, Greenwich, CT, 1994, vol. 2B, pp. $117-186$

33 L. M. Delves, Nucl. Phys., 1959, 9, 391; 1960, 20, 275.

34 This is a straight-forward extension to three- dimensions of the bi-cubic spline procedure described by W. H. Press, B. P. Flannery, S. A. Teukolsky and W. T. Vetterling, in Numerical Recipes, Cambridge University Press, Cambridge, 1989, pp. 100-101.

35 W. Kolos, K. Szalewicz and H. J. Monkhorst, J. Chem. Phys., 1986, 84, 3278.

36 H. Partridge, C. W. Bauschlicher, J. R. Sallcop and E. Levin, J. Chem. Phys., 1993, 99, 5951.

37 The Linpack Benchmark, TOP500, http://www.netlib.org/ benchmark/top500/top500.list.html, 18 June 1998.

38 A. Kuppermann and Y-S. M. Wu, to be published.

39 Ref. 15, p. 427.

40 N. C. Handy and A. M. Lee, Chem. Phys. Lett., 1996, 252, 425.

41 W. Kutzelnigg, Mol. Phys., 1997, 90, 909.

42 W. Cencek, J. Rychlewski, R. Jaquet and W. Kutzelnigg, J. Chem. Phys., 1998, 108, 2831.

43 R. Jaquet, W. Cencek, W. Kutzelnigg and J. Rychlewski, J. Chem. Phys., 1998, 108, 2837.

44 L. Wolniewicz, J. Chem. Phys., 1993, 99, 1851.

Paper $8 / 08797$ K 